\title{
Intuitionistic fuzzy action of a group on a set
}

\author{
Sinem Tarsuslu (Yılmaz) ${ }^{1}$, Ali Tarsuslu ${ }^{2}$ and Mehmet Çitil ${ }^{3}$ \\ ${ }^{1}$ Mersin University, Faculty of Arts and Sciences \\ Department of Mathematics \\ Mersin, Turkey \\ e-mail: sinemnyilmaz@gmail.com \\ ${ }^{2}$ Private Science Academy Children's Club, Mersin, Turkey \\ e-mail: alitarsuslu@gmail .com \\ ${ }^{3}$ Kahramanmaraş Sütçü İmam University \\ Faculty of Arts and Sciences, Department of Mathematics \\ Kahramanmaraş, Turkey \\ e-mail: mehmet.citil4600@gmail.com
}

Received: 27 March 2018

Accepted: 20 April 2018

\begin{abstract}
Intuitionistic fuzzy set theory was introduced by Atanassov as an extension of fuzzy sets [1]. The algebraic structures like groups, rings, modules, etc. were generalized to intuitionistic fuzzy sets by different authors. Some properties of them were studied $[4,9,11,14,15]$. In this study, we generalized the action of a group on a set to intuitionistic fuzzy action. We obtained some basic results.
\end{abstract}

Keywords: Intuitionistic fuzzy sets, Intuitionistic fuzzy algebraic structures, Intuitionistic fuzzy actions.

2010 Mathematics Subject Classification: 03E72, 47S40.

\section{Introduction}

The intuitionistic fuzzy set theory was introduced by Atanassov in 1983 [1,2] as an extension of fuzzy sets by enlarging the truth value set to the lattice $[0,1] \times[0,1]$ is defined as following.

Definition 1. Let $L=[0,1]$ then

$$
L^{*}=\left\{\left(x_{1}, x_{2}\right) \in[0,1]^{2}: x_{1}+x_{2} \leq 1\right\}
$$


is a lattice with $\left(x_{1}, x_{2}\right) \leq\left(y_{1}, y_{2}\right): \Longleftrightarrow " x_{1} \leq y_{1}$ and $x_{2} \geq y_{2}$ ".

For $\left(x_{1}, y_{1}\right),\left(x_{2}, y_{2}\right) \in L^{*}$,the operators $\wedge$ and $\vee$ on $\left(L^{*}, \leq\right)$ are defined as follows: $\left(x_{1}, y_{1}\right) \wedge$ $\left(x_{2}, y_{2}\right)=\left(\min \left(x_{1}, x_{2}\right), \max \left(y_{1}, y_{2}\right)\right)\left(x_{1}, y_{1}\right) \vee\left(x_{2}, y_{2}\right)=\left(\max \left(x_{1}, x_{2}\right), \min \left(y_{1}, y_{2}\right)\right)$ For each $J \subseteq L^{*}$

$$
\sup J=(\sup \{x:(x, y \in[0,1]),((x, y) \in J)\}, \inf \{y:(x, y \in[0,1])((x, y) \in J)\})
$$

and

$$
\inf J=(\inf \{x:(x, y \in[0,1])((x, y) \in J)\}, \sup \{y:(x, y \in[0,1])((x, y) \in J)\}) .
$$

Definition 2 ([1]). An intuitionistic fuzzy set (shortly IFS) on a set X is an object of the form

$$
A=\left\{\left\langle x, \mu_{A}(x), \nu_{A}(x)\right\rangle: x \in X\right\}
$$

where $\mu_{A}(x),\left(\mu_{A}: X \rightarrow[0,1]\right)$ is called the "degree of membership of $x$ in $A$ ", $\nu_{A}(x),\left(\nu_{A}\right.$ : $X \rightarrow[0,1])$ is called the " degree of non-membership of $x$ in $A$ ", and where $\mu_{A}$ and $\nu_{A}$ satisfy the following condition:

$$
\mu_{A}(x)+\nu_{A}(x) \leq 1, \text { for all } x \in X .
$$

The hesitation degree of $x$ is defined by $\pi_{A}(x)=1-\mu_{A}(x)-\nu_{A}(x)$.

Definition 3 ([1]). An IFS $A$ is said to be contained in an IFS $B$ (notation $A \sqsubseteq B$ ) if and only if, for all $x \in X: \mu_{A}(x) \leq \mu_{B}(x)$ and $\nu_{A}(x) \geq \nu_{B}(x)$.

It is clear that $A=B$ if and only if $A \sqsubseteq B$ and $B \sqsubseteq A$.

Definition 4 ([1]). Let $A \in I F S$ and let $A=\left\{\left\langle x, \mu_{A}(x), \nu_{A}(x)\right\rangle: x \in X\right\}$ then the above set is called the complement of $A$

$$
A^{c}=\left\{\left\langle x, \nu_{A}(x), \mu_{A}(x)\right\rangle: x \in X\right\}
$$

The concept of intuitionistic fuzzy relation was introduced by Burillo and Bustince. Some fundamental properties were studied in $[6,7]$.

Definition 5 ([6]). An intuitionistic fuzzy relation(shortly IFR) is an intuitionistic fuzzy subset of $X \times Y$, that is, is an expression $R$ given by $R=\left\{\left\langle(x, y), \mu_{R}(x, y), \nu_{R}(x, y)\right\rangle: x \in X, y \in Y\right\}$ where $\mu_{R}: X \times Y \rightarrow[0,1], \nu_{R}: X \times Y \rightarrow[0,1]$ satisfy the condition $0 \leq \mu_{R}(x, y)+\nu_{R}(x, y) \leq 1$ for any $(x, y) \in X \times Y$.

Definition 6 ([13]). Let $X$ be a non-empty set and $R \in I F R(X)$.

ER1 For every $x \in X$,

$$
\begin{aligned}
& \mu_{R}(x, x)=1 \\
& \nu_{R}(x, x)=0
\end{aligned}
$$

then $R$ is called an intuitionistic fuzzy reflexive. 
ER2 For every $x, y \in X$,

$$
\begin{gathered}
\mu_{R}(x, y) \leq \mu_{R}(y, x) \\
\nu_{R}(x, y) \geq \nu_{R}(y, x)
\end{gathered}
$$

then $R$ is called an intuitionistic fuzzy symmetric.

ER3 For every $x, y, z \in X$,

$$
\begin{array}{r}
\mu_{R}(x, y) \wedge \mu_{R}(y, z) \leq \mu_{R}(x, z) \\
\nu_{R}(x, y) \vee \nu_{R}(y, z) \geq \nu_{R}(x, z)
\end{array}
$$

then $R$ is called an intuitionistic fuzzy transitive.

If an intuitionistic fuzzy relation satisfies the previous properties then it is called an intuitionistic fuzzy equivalence relation $(I F E(X))$.

Now we can talk about intuitionistic fuzzy equivalence classes of $R$.

Definition 7 ([13]). Let $X$ be a non-empty set, $R \in I F E(X)$ and $a \in X$.

$$
[a]_{R}=\left\{\left\langle x, \mu_{[a]_{R}}(x), \nu_{[a]_{R}}(x)\right\rangle: x \in X\right\}
$$

where $\mu_{[a]_{R}}(x)=\mu_{R}(a, x), \nu_{[a]_{R}}(x)=\nu_{R}(a, x)$ is called an intuitionistic fuzzy equivalence class of a w.r.t R.

Intuitionistic fuzzy group theory has been a subject of interest to several researchers. In this study, we will examined the concept of group action an a set for intuitionistic fuzzy sets. Let's firstly remember the definitions of intuitionistic fuzzy group and crisp act.

Definition 8 ([4]). Let $G$ be a group, $A \in I F S(G)$. If for all $x, y \in G$,

1. $A(x y) \geq \min (A(x), A(y))$

2. $A\left(x^{-1}\right) \geq A(x)$

then A called an intuitionistic fuzzy subgroup over $G$, shortly $I F G(G)$.

Definition 9 ([10]). Let $G$ be a group and $S$ a non-empty set. $\cdot: G \times S \rightarrow S$ is an action of $G$ on $S$ if and only if for all $g, h \in G, x \in S$

1. $(g h) x=h(g x)$

2. $e x=x$ 


\section{Intuitionistic fuzzy actions}

The algebraic structures were generalized using Zadeh [16] extension principle by several authors. The concept of actions of a group fuzzified by Boixader and Recasens [5]. In this study, we introduced intuitionistic fuzzy actions of a group on a set by extending definition to the lattice $[0,1] \times[0,1]$.

Definition 10. Let $G$ be a group, $S$ a non-empty set and $\theta$ be a crisp action of $G$ on $S$.

$$
\bar{\alpha}: G \times S_{G} \rightarrow[0,1] \times[0,1]
$$

such that $\operatorname{Im} \theta=S_{G}$ and $\bar{\alpha}\left(s_{g}, s^{\prime}\right)=\left\{<\left(s_{g}, s^{\prime}\right), \mu_{\bar{\alpha}}\left(s_{g}, s^{\prime}\right), \nu_{\bar{\alpha}}\left(s_{g}, s^{\prime}\right)>:\left(s_{g}, s^{\prime}\right) \in G \times S_{G}\right\}$ is an intuitionistic fuzzy action of $G$ on $S$ if and only if for all $g, h \in G, s \in S$

1. $\bar{\alpha}\left(s_{h g}, y\right) \wedge \bar{\alpha}\left(s_{g}, s^{\prime}\right) \leq \bar{\alpha}\left(s_{h}^{\prime}, s^{\prime \prime}\right)$

2. $\bar{\alpha}\left(s_{g}, s^{\prime}\right) \wedge \bar{\alpha}\left(s_{h}^{\prime}, s^{\prime \prime}\right) \leq \bar{\alpha}\left(s_{h g}, s^{\prime \prime}\right)$

3. $\bar{\alpha}\left(s_{e}, s\right)=(1,0)$

$\bar{\alpha}\left(s_{g}, s^{\prime}\right)$ is the degree of $s^{\prime}$ which is the image of $s$ under the action of $g$.

Lemma 1. Let $G$ be a group, $S$ a non-empty set and $\bar{\alpha}$ be an intuitionistic fuzzy action of $G$ on $S$. For $g \in G$ and $s, s^{\prime} \in S$,

$$
\bar{\alpha}\left(s_{g}, s^{\prime}\right)=\bar{\alpha}\left(s_{g^{-1}}^{\prime}, s\right) .
$$

Proof. It is clear that:

$$
\begin{aligned}
\mu_{\bar{\alpha}}\left(s_{e}, s\right) \wedge \mu_{\bar{\alpha}}\left(s_{g}, s^{\prime}\right) & \leq \mu_{\bar{\alpha}}\left(s_{g^{-1}}^{\prime}, s\right) \\
\mu_{\bar{\alpha}}\left(s_{e}, s\right) \wedge \mu_{\bar{\alpha}}\left(s_{g^{-1}}^{\prime}, s\right) & \leq \mu_{\bar{\alpha}}\left(s_{g}, s^{\prime}\right)
\end{aligned}
$$

and

$$
\begin{gathered}
\nu_{\bar{\alpha}}\left(s_{e}, s\right) \vee \nu_{\bar{\alpha}}\left(s_{g}, s^{\prime}\right) \geq \nu_{\bar{\alpha}}\left(s_{g^{-1}}^{\prime}, s\right) \\
\nu_{\bar{\alpha}}\left(s_{e}, s\right) \vee \nu_{\bar{\alpha}}\left(s_{g^{-1}}^{\prime}, s\right) \geq \nu_{\bar{\alpha}}\left(s_{g}, s^{\prime}\right)
\end{gathered}
$$

Example 1. Let $G$ be a group and $H$ be a subgroup of $G$. An intuitionistic fuzzy action of $H$ on $G$ is given by $\bar{\alpha}\left(s_{h}, h s h^{-1}\right)$ called intuitionistic fuzzy conjugation by $h$.

Proposition 1. Let $G$ be a group, $S$ a non-empty set and $\bar{\alpha}$ be an intuitionistic fuzzy action of $G$ on $S$. E is intuitionistic fuzzy equivalence relation on $S$ defined for all $s, s^{\prime} \in S, E\left(s, s^{\prime}\right)=$ $\bar{\alpha}\left(s_{e}, s^{\prime}\right)$.

Proof. (i) Since $\mu_{\bar{\alpha}}\left(s_{e}, s\right)=1$ and $\nu_{\bar{\alpha}}\left(s_{e}, s\right)=0$ then $E(s, s)=(1,0)$.

(ii) From Lemma 1 we obtain that

$$
\mu_{\bar{\alpha}}\left(s_{e}, s^{\prime}\right)=\mu_{\bar{\alpha}}\left(s_{e}^{\prime}, s\right)
$$


and

$$
\nu_{\bar{\alpha}}\left(s_{e}, s^{\prime}\right)=\nu_{\bar{\alpha}}\left(s_{e}^{\prime}, s\right) .
$$

So, $E\left(s, s^{\prime}\right)=E\left(s^{\prime}, s\right)$.

(iii) It is clear form definition,

$$
\mu_{\bar{\alpha}}\left(s_{e}, s^{\prime}\right) \wedge \mu_{\bar{\alpha}}\left(s_{e}^{\prime}, s^{\prime \prime}\right) \leq \mu_{\bar{\alpha}}\left(s_{e}, s^{\prime \prime}\right)
$$

and

$$
\nu_{\bar{\alpha}}\left(s_{e}, s^{\prime}\right) \vee \nu_{\bar{\alpha}}\left(s_{e}^{\prime}, s^{\prime \prime}\right) \geq \nu_{\bar{\alpha}}\left(s_{e}, s^{\prime \prime}\right) .
$$

Theorem 1. Let $G$ be a group, $S$ a non-empty set and $\bar{\alpha}$ be an intuitionistic fuzzy action of $G$ on $S$.

1. $R_{\bar{\alpha}}\left(s, s^{\prime}\right)=\sup _{g \in G} \bar{\alpha}\left(s_{g}, s^{\prime}\right)$, for all $s, s^{\prime} \in S$ is an intuitionistic fuzzy equivalence relation on $S$.

2. For all fixed $s \in S, G_{s}=\left\{\left\langle g, \mu_{\bar{\alpha}}\left(s_{g}, s\right), \nu_{\bar{\alpha}}\left(s_{g}, s\right)\right\rangle: g \in G\right\}$ is an intuitionistic fuzzy subgroup of $G$.

Proof. (1)Since $\sup _{g \in G} \bar{\alpha}\left(s_{g}, s\right) \geq \bar{\alpha}\left(s_{e}, s^{\prime}\right)=(1,0)$ then $R_{\bar{\alpha}}(s, s)=(1,0)$ for all $s \in S$.

$$
\begin{aligned}
R_{\bar{\alpha}}\left(s, s^{\prime}\right)=\sup _{g \in G} \bar{\alpha}\left(s_{g}, s^{\prime}\right) & =\sup _{g \in G} \bar{\alpha}\left(s_{g^{-1}}^{\prime}, s\right)=R_{\bar{\alpha}}\left(s^{\prime}, s\right) \text { for all } s, s^{\prime} \in S . \\
R_{\bar{\alpha}}\left(s, s^{\prime}\right) \wedge R_{\bar{\alpha}}\left(s^{\prime}, s^{\prime \prime}\right)= & \left(\sup _{g \in G} \bar{\alpha}\left(s_{g}, s^{\prime}\right)\right) \wedge\left(\sup _{h \in G} \bar{\alpha}\left(s_{h}^{\prime}, s^{\prime \prime}\right)\right) \\
& =\sup _{g, h \in G}\left(\bar{\alpha}\left(s_{g}, s^{\prime}\right) \wedge \bar{\alpha}\left(s_{h}^{\prime}, s^{\prime \prime}\right)\right) \\
& \leq \sup _{g, h \in G} \bar{\alpha}\left(s_{h g}, s^{\prime \prime}\right)=R_{\bar{\alpha}}\left(s, s^{\prime \prime}\right) \text { for all } s, s^{\prime}, s^{\prime \prime} \in S .
\end{aligned}
$$

(2) $\mu_{\bar{\alpha}}\left(s_{e}, s\right)=1 \wedge \nu_{\bar{\alpha}}\left(s_{e}, s\right)=0$, for trivial element $e$.

Let $g, h \in G$,

$$
\mu_{\bar{\alpha}}\left(s_{h g}, s\right) \geq \mu_{\bar{\alpha}}\left(s_{g}, s\right) \wedge \mu_{\bar{\alpha}}\left(s_{h}, s\right)
$$

and

$$
\nu_{\bar{\alpha}}\left(s_{h g}, s\right) \leq \nu_{\bar{\alpha}}\left(s_{g}, s\right) \vee \nu_{\bar{\alpha}}\left(s_{h}, s\right)
$$

So, $G_{s}(g h) \geq G_{s}(g) \wedge G_{s}(h)$.

Let $g \in G$,

$$
\mu_{\bar{\alpha}}\left(s_{g}, s\right)=\mu_{\bar{\alpha}}\left(s_{g^{-1}}, s\right)
$$

and

$$
\nu_{\bar{\alpha}}\left(s_{g}, s\right)=\nu_{\bar{\alpha}}\left(s_{g^{-1}}, s\right)
$$

Hence, $G_{s}(g)=G_{s}\left(g^{-1}\right)$.

Definition 11. Let $G$ be a group, $S$ a non-empty set and $\bar{\alpha}$ be an intuitionistic fuzzy action of $G$ on $S$. The intuitionistic fuzzy equivalence class of $s \in S$ w.r.t $R_{\bar{\alpha}}$ is called intuitionistic fuzzy orbit of $s$. 
Example 2. If $\bar{\alpha}$ an intuitionistic fuzzy action of $G$ on itself then the intuitionistic fuzzy orbit of $x \in G, A_{s}=\left\{g s g^{-1}: g \in G\right\}$ called intuitionistic fuzzy conjugacy class of $s$.

$$
\nu_{\bar{\alpha}}(e, x, y) \vee \nu_{\bar{\alpha}}(e, y, z) \geq \nu_{\bar{\alpha}}(e, x, z) .
$$

\section{Conclusion}

The concept of intuitionistic fuzzy action can be used on Sylow theorems and other structure theorems for groups for intuitionistic fuzzy set theory. Also, intuitionistic fuzzy action will be studied on modal operators. Intuitionistic fuzzy modal operators have interested several authors $[3,8,12]$.

\section{Acknowledgement}

This study was supported by the Research Fund of Mersin University in Turkey with Project Number: 2017-2-AP2-2616.

\section{References}

[1] Atanassov, K. T. (1983) Intuitionistic Fuzzy Sets, VII ITKR Session, Sofia, June, 1983 (Deposed in Centr. Sci.-Techn. Library of the Bulg. Acad. of Sci., 1697/84) (in Bulgarian). Reprinted: Int. J. Bioautomation, 2016, 20 (S1), S1-S6.

[2] Atanassov, K. T. (1999) Intuitionistic Fuzzy Sets, Spinger, Heidelberg.

[3] Atanassov, K. T., Çuvalcıŏglu G., \& Atanassova, V. (2014) A new modal operator over intuitionistic fuzzy sets, Notes on Intuitionistic Fuzzy Sets, 20(5), 1-8.

[4] Biswas, R. (1989) Intuitionistic fuzzy subgroups, Mathematical Forum, Vol. X, 37-46.

[5] Boixader, D., \& Recasens, J. (2018) Fuzzy Actions, Fuzzy Sets and Systems, 339, 17-30.

[6] Burillo, P., \& Bustince, H. (1995) Intuitionistic fuzzy relations (Part I) Mathware \& Soft Computing, 2, 5-38.

[7] Bustince, H., \& Burillo, P. (1996) Structures on intuitionistic fuzzy relations, Fuzzy Sets and Systems, 78, 293-303.

[8] Çuvalcıŏglu, G. (2010) Expand the modal operator diagram with $Z_{\alpha, \beta}^{\omega}$, Proc. Jangjeon Math. Soc, 13 (3), 403-412.

[9] Çuvalcıŏ̆lu, G., \& Tarsuslu (Yılmaz), S. (2017) Universal algebra in intuitionistic fuzzy set theory, Notes on Intuitionistic Fuzzy Sets, 23(1), 1-5.

[10] Hungerford, T. W. (1974) Algebra, Springer, Verlag, New York Inc. 
[11] Hur, K., Jang Y. \& Kang H. W. (2003) Intuitionistic fuzzy Subgroupoid, Int. Jour. of Fuzzy Logic and Int. Sys., 3(1), 72-77.

[12] Yılmaz, S., Çuvalcıŏ̆lu G., (2014) On level operators for temporal intuitionistic fuzzy sets, Notes on Intuitionistic Fuzzy Sets, 20(2), 6-15.

[13] Hur, K., Jang, S. Y. \& Ahn, Y. S. (2005) Intuitionistic fuzzy equivalence relations, Honam Math. J. 27(2), 163-181.

[14] Yılmaz, S., Çuvalcıoğlu G. (2016). On Study of Some Intuitionistic Fuzzy Operators for Intuitionistic Fuzzy Algebraic Structures, Journal of Fuzzy Set Valued Analysis, 2016(3), $317-325$.

[15] Yan, L., (2008) Intuitionistic Fuzzy Ring and Its Homomorphism Image, Int. Seminar on FBM Inf. Eng., 75-77.

[16] Zadeh, L.A. (1965) Fuzzy Sets, Information and Control, 8, 338-353. 